\title{
Role of photodynamic therapy using talaporfin sodium and a semiconductor laser in patients with newly diagnosed glioblastoma
}

\author{
Masayuki Nitta, MD, PhD, ,,2 Yoshihiro Muragaki, MD, PhD, ${ }^{1,2}$ Takashi Maruyama, MD, PhD, 1,2 \\ Hiroshi Iseki, MD, PhD, ${ }^{2}$ Takashi Komori, MD, PhD, ${ }^{3}$ Soko Ikuta, PhD, ${ }^{2}$ Taiichi Saito, MD, PhD, \\ Takayuki Yasuda, MD, ${ }^{1}$ Junji Hosono, MD, PhD, ${ }^{1}$ Saori Okamoto, MD, PhD, ${ }^{1}$ \\ Shunichi Koriyama, MD, ${ }^{1}$ and Takakazu Kawamata, MD, PhD'
}

\begin{abstract}
${ }^{1}$ Department of Neurosurgery and ${ }^{2}$ Faculty of Advanced Techno-Surgery, Institute of Biomedical Engineering and Science, Tokyo Women's Medical University; and ${ }^{3}$ Department of Laboratory Medicine and Pathology (Neuropathology), Tokyo Metropolitan Neurological Hospital, Tokyo, Japan
\end{abstract}

\begin{abstract}
OBJECTIVE In this study on the effectiveness and safety of photodynamic therapy (PDT) using talaporfin sodium and a semiconductor laser, the long-term follow-up results of 11 patients with glioblastoma enrolled in the authors' previous phase II clinical trial (March 2009-2012) and the clinical results of 19 consecutive patients with newly diagnosed glioblastoma prospectively enrolled in a postmarket surveillance (March 2014-December 2016) were analyzed and compared with those of 164 patients treated without PDT during the same period.
\end{abstract}

METHODS The main outcome measures were the median overall survival (OS) and progression-free survival (PFS) times. Moreover, the adverse events and radiological changes after PDT, as well as the patterns of recurrence, were analyzed and compared between the groups. Kaplan-Meier curves were created to assess the differences in OS and PFS between the groups. Univariate and multivariate analyses were performed to identify the prognostic factors, including PDT, among patients with newly diagnosed glioblastoma.

RESULTS The median PFS times of the PDT and control groups were 19.6 and 9.0 months, with 6-month PFS rates of $86.3 \%$ and $64.9 \%$, respectively $(p=0.016)$. The median OS times were 27.4 and 22.1 months, with 1 -year OS rates of $95.7 \%$ and $72.5 \%$, respectively $(p=0.0327$ ). Multivariate analyses found PDT, preoperative Karnofsky Performance Scale score, and IDH mutation to be significant independent prognostic factors for both OS and PFS. Eighteen of 30 patients in the PDT group experienced tumor recurrence, including local recurrence, distant recurrence, and dissemination in 10, 3, and 4 patients, respectively. Conversely, 141 of 164 patients in the control group experienced tumor recurrence, including 101 cases of local recurrence. The rate of local recurrence tended to be lower in the PDT group $(p=0.06)$.

CONCLUSIONS The results of the present study suggest that PDT with talaporfin sodium and a semiconductor laser provides excellent local control, with few adverse effects even in cases of multiple laser irradiations, as well as potential survival benefits for patients with newly diagnosed glioblastoma.

https://thejns.org/doi/abs/10.3171/2018.7.JNS18422

KEYWORDS photodynamic therapy; talaporfin sodium; semiconductor laser; glioblastoma; newly diagnosed; oncology

$\mathrm{E}$ FFECTIVE glioblastoma treatment represents a major unmet medical need. The current standard treatment for glioblastoma, which includes resection followed by radiation therapy and temozolomide-based chemotherapy, results in a median survival of less than 15 months. ${ }^{21} \mathrm{~A}$ greater extent of resection is associated with better survival; $6,9,18$ however, due to its infiltrate nature of the lesion, complete elimination of tumor cells is generally impossible, and glioblastomas hence recur in most patients. Thus, development of new therapeutics is necessary for this malignant tumor.

Photodynamic therapy (PDT) is defined as the admin-

ABBREVIATIONS CTCAE = Common Terminology Criteria for Adverse Events; KPS = Karnofsky Performance Scale; MGMT = 0-6-methylguanine-DNA methyltransferase; OS = overall survival; PDT = photodynamic therapy; PFS = progression-free survival.

SUBMITTED February 13, 2018. ACCEPTED July 24, 2018.

INCLUDE WHEN CITING Published online December 7, 2018; DOI: 10.3171/2018.7.JNS18422. 
istration of a photosensitizer, a nontoxic chemical, to a patient bearing a lesion via illumination of the lesion with light; in the presence of oxygen, this leads to the generation of cytotoxic species such as singlet oxygen and, consequently, to cell death. ${ }^{1,5,17}$

PDT for cancer treatment was first established in the 1960s, and a hematoporphyrin derivative known as photophyrin was used as the first PDT agent for several types of cancer, including lung, esophageal, stomach, and cervical cancers., ${ }^{711,16}$ Talaporfin sodium (mono-L-aspartyl chlorine e6 or NPe6) is a second-generation photosensitizer for PDT. Its administration in combination with a semiconductor laser has been approved for various cancers, including lung cancer $^{7}$ (2003), malignant brain tumors ${ }^{14}$ (2013), and esophageal cancer ${ }^{28}$ (2015), in Japan.

Malignant glioma may be a good candidate for PDT, for the following reasons. First, although the extent of tumor resection correlates with the prognosis in patients with glioblastoma, it is difficult to achieve both maximum tumor resection and preservation of brain function, because the tumors are often extended to, or are located in, the eloquent region of the brain. PDT, however, is expected to selectively kill the infiltrating tumor cells without damaging the surrounding normal brain cells. Second, despite its infiltrating nature, local recurrence is the major pattern of glioblastoma relapse, even after gross-total removal of the enhanced lesion, ${ }^{8,15,19}$ and local control of the tumor, as well as systemic treatments, is thus essential.

A phase II clinical trial for PDT with talaporfin sodium and a semiconductor laser was conducted between 2009 and 2012 in our institute and Tokyo Medical University; the study based on that trial showed the safety of the treatment and excellent clinical results for patients with newly diagnosed glioblastoma. ${ }^{14}$ The median progression-free survival (PFS) and overall survival (OS) times for 13 patients with newly diagnosed glioblastoma were 12.0 and 24.8 months after treatment, respectively. ${ }^{14}$ That study also revealed that PDT may provide excellent local control, showing a local PFS - that is, PFS of the laser-irradiated sites - of 19.6 months..$^{14}$ Given the favorable clinical results of that phase II study, PDT for malignant brain tumors was approved in Japan in September 2013. Subsequently, our institute joined a prospective postmarket surveillance of patients with newly diagnosed glioblastoma treated with PDT in 2014. In the present study, we report the clinical results of this postmarket surveillance together with the long-term follow-up results of the patients with newly diagnosed glioblastoma enrolled in the previous phase II clinical trial. We also compare the clinical results of these patients to those of patients with newly diagnosed glioblastoma who underwent surgical treatment without PDT during the same period.

\section{Methods \\ Patient Selection}

The long-term follow-up results of 11 patients enrolled in the phase II clinical trial (March 2009-2012) in our institute $^{14}$ and the clinical results of 19 consecutive patients with newly diagnosed glioblastoma prospectively enrolled in the postmarket surveillance between March 2014 and
December 2016 were analyzed. The primary endpoint of the prospective study was OS time and adverse effects. The secondary endpoint was PFS time. This study was approved by the institutional review board, and informed consent was obtained from all study patients. The clinical results of these patients were retrospectively compared with those of 164 consecutive patients with newly diagnosed glioblastoma who were treated without PDT between March 2009 and December 2016. To avoid selection bias, patients who underwent biopsy were excluded.

\section{Surgical Treatment and PDT}

Patients who were treated with PDT received a single intravenous injection of talaporfin sodium (Laserphyrin, Meiji Seika Pharma Co.,Ltd.) at a dose of $40 \mathrm{mg} / \mathrm{m}^{2}, 22-26$ hours prior to undergoing tumor removal. All patients underwent maximum removal of the enhanced lesion. After resection, irradiation of the resection cavity with a 664-nm semiconductor laser beam (Meiji Seika Pharma Co., Ltd.) (diameter $1.5 \mathrm{~cm}$, radiation power density $150 \mathrm{~mW} / \mathrm{cm}^{2}$, and radiation energy density $27 \mathrm{~J} / \mathrm{cm}^{2}$ ) was performed in patients in the PDT group. Particular emphasis was placed on irradiation of the areas at a high risk of recurrence, such as the genu of the corpus callosum and deep white matter. In the phase II clinical study, the number of laser irradiations was limited to up to 3 spots in consideration of the safety of the patients. However, there was no limitation in the irradiation spots after the approval of the treatment; therefore, multiple laser irradiations were subsequently performed, without limitations of the number, to ensure that the entire resection cavity wall was covered. Each irradiation produced a circular lesion $1.5 \mathrm{~cm}$ in diameter, and irradiation was performed without overlap of the irradiated area. In all cases, laser irradiation was performed 22-26 hours after administration of talaporfin sodium.

Postoperatively, all patients received fractionated radiation therapy (total $60 \mathrm{~Gy}$ ) and concomitant temozolomidebased chemotherapy, along with at least 6 courses of maintenance temozolomide-based chemotherapy, except for patients with early recurrence.

\section{Histological and Molecular Diagnoses}

Histological diagnosis was performed according to the 2007 WHO guidelines..$^{10}$ IDH mutation status was examined based on immunohistochemistry using an R132Hspecific antibody (dianova $\mathrm{GmbH}$ ); if it was negative, direct sequence of the tumor DNA was performed. The presence of $1 \mathrm{p} / 19 \mathrm{q}$ codeletion was analyzed based on fluorescence in situ hybridization. $O$-6-methylguanine-DNA methyltransferase (MGMT) methylation status was not analyzed; however, the expression of the MGMT protein was evaluated based on immunohistochemistry.

\section{Volumetric Analysis}

Tumor volumes, as determined from axial contrastenhanced lesion on T1-weighted MRI, were calculated by importing DICOM images from the MRI unit to Leksell GammaPlan software (Elekta). Extent of resection was calculated based on the following: (preoperative tumor volume - postoperative tumor volume)/preoperative tumor 
volume. The volumetric analysis was performed by a neurosurgeon blinded to patient information.

\section{Patient Outcomes}

Clinical data were collected from the patient records and telephone interviews. The two main outcome measures were the median OS and PFS times. OS was defined as the time between the initial surgery and death, and the PFS was defined as the time between the initial surgery and the demonstration of an unequivocal increase in an enhanced lesion on follow-up imaging or as obvious clinical deterioration. The Response Assessment in Neuro-Oncology Criteria were used for radiological assessment. ${ }^{27}$ Patients with no known progression were censored as of their last visit and/or scan date. Toxicity was examined retrospectively by inspecting the patients' records. The severity of adverse effects was evaluated according to the Common Terminology Criteria for Adverse Events (CTCAE) version 4.0.

\section{Statistical Analysis}

The OS and PFS were estimated based on the Kaplan-Meier method. The log-rank test was used to evaluate the importance of prognostic factors that might affect survival. Data analysis was performed using the JMP statistical software (SAS Inc.). Univariate analyses for OS and PFS were performed using Cox proportional hazards modeling. Variables that were statistically significant in the univariate analyses were further analyzed using multivariate analyses. Hazard ratios and $95 \%$ confidence intervals are reported, along with the 2-tailed probability values. The reported probability values in the Cox model are based on the Wald test, and values $<0.05$ were considered significant.

\section{Results}

\section{Patient Characteristics}

The characteristics of the study patients are summarized in Table 1. The PDT group consisted of 30 patients (15 males and 15 females), with a median age at disease onset of 52 years (range 24-79 years). The control group comprised 164 patients (103 males and 61 females), with a median age at disease onset of 61 years (range 21-77 years). There was no statistical difference in the age of the patients between the two groups $(\mathrm{p}=0.648)$. The distribution of the Karnofsky Performance Scale (KPS) scores before surgery was similar in the two groups $(\mathrm{p}=0.13)$. The median preoperative tumor volumes in the PDT and control groups were $39.5 \mathrm{~cm}^{3}$ and $38.5 \mathrm{~cm}^{3}$, respectively, and there was no statistically significant difference between the two groups $(p=0.31)$. All patients underwent maximum resection of the enhanced lesion, and those in the PDT group received subsequent laser irradiation for the resection cavity wall. The median number of laser irradiation spots was 3 (range 1-13). The mean extent of resection of the enhanced lesion in the PDT and control groups was $97.2 \%$ and $97.7 \%$, respectively $(\mathrm{p}=$ 0.37 ). Furthermore, there were no differences in the histological diagnoses of the removed tumors between the two groups. IDH mutation was noted in $13.0 \%$ and $15.0 \%$
TABLE 1. Patient characteristics

\begin{tabular}{|c|c|c|c|}
\hline Characteristic & PDT & Control & $\mathrm{p}$ Value \\
\hline No. of patients & 30 & 164 & \\
\hline \multicolumn{4}{|l|}{ Age } \\
\hline Mean \pm SD & $54.1 \pm 2.8$ & $55.4 \pm 1.2$ & 0.648 \\
\hline Median (range) & $52(24-79)$ & $61(21-77)$ & \\
\hline Sex, no. & & & 0.19 \\
\hline Male & 15 & 103 & \\
\hline Female & 15 & 61 & \\
\hline $\begin{array}{l}\text { Median preop KPS score } \\
\quad \text { (range) }\end{array}$ & $80(30-100)$ & $80(30-100)$ & 0.13 \\
\hline Preop tumor vol, $\mathrm{cm}^{3}$ & & & 0.31 \\
\hline Mean \pm SD & $52.6 \pm 7.2$ & $44.1 \pm 4.2$ & \\
\hline Median (range) & $39.5(7.2-217)$ & $38.5(2.0-83.2)$ & \\
\hline \multicolumn{4}{|l|}{$\mathrm{EOR}, \%$} \\
\hline Mean \pm SD & $97.2 \pm 0.5$ & $97.7 \pm 0.21$ & 0.37 \\
\hline Median (range) & $98.0(90-100)$ & $98.0(89-100)$ & \\
\hline Histology (WHO 2007) & & & 0.57 \\
\hline Glioblastoma & $21(70.0 \%)$ & $126(76.8 \%)$ & \\
\hline GBMO & $8(26.7 \%)$ & $36(22.0 \%)$ & \\
\hline Gliosarcoma & $1(3.3 \%)$ & $1(0.6 \%)$ & \\
\hline \multicolumn{4}{|l|}{ MIB-1 index } \\
\hline Mean \pm SD & $26.7 \pm 1.9$ & $20.4 \pm 0.9$ & 0.003 \\
\hline Median (range) & $16.5(10.1-53)$ & $16.1(2.1-58)$ & \\
\hline Rate of IDH mutation & $13.0 \%$ & $15.0 \%$ & 0.8 \\
\hline MGMT protein expression & & & 0.14 \\
\hline High & $6(25.0 \%)$ & $59(43.7 \%)$ & \\
\hline Low & $4(16.7 \%)$ & $10(7.4 \%)$ & \\
\hline None & $14(58.3 \%)$ & $66(48.9 \%)$ & \\
\hline
\end{tabular}

EOR = extent of resection; $\mathrm{GBMO}$ = glioblastoma multiforme with oligodendroglial component.

of patients in the PDT and control groups, respectively $(p=0.8)$. There was no $1 p / 19 q$ codeletion in any patient. The MGMT protein expression did not differ between the groups $(\mathrm{p}=0.14)$. In fact, the only patient characteristic that significantly differed between the groups was the MIB-1 index; the PDT group exhibited a higher mean MIB-1 index $(26.7 \pm 1.9)$ than the control group (20.4 \pm $0.9 ; \mathrm{p}=0.003)($ Table 1$)$.

\section{Prognosis}

The median PFS time and 6-month PFS rate of the 30 patients with newly diagnosed glioblastomas treated with PDT were 19.6 months and $86.3 \%$, respectively. The median PFS time and 6-month PFS rate of the control group were 9.0 months and $64.9 \%$, respectively. The PDT group showed significantly longer PFS $(\mathrm{p}=0.016$; Fig. 1A). In the PDT group, the median OS time was 27.4 months, and the 1-year OS rate was $95.7 \%$, while in the control group, the corresponding values were 22.1 months and $72.5 \%$, respectively; the PDT group showed significantly better OS than the control group $(\mathrm{p}=0.0327$; Fig. 1B). 

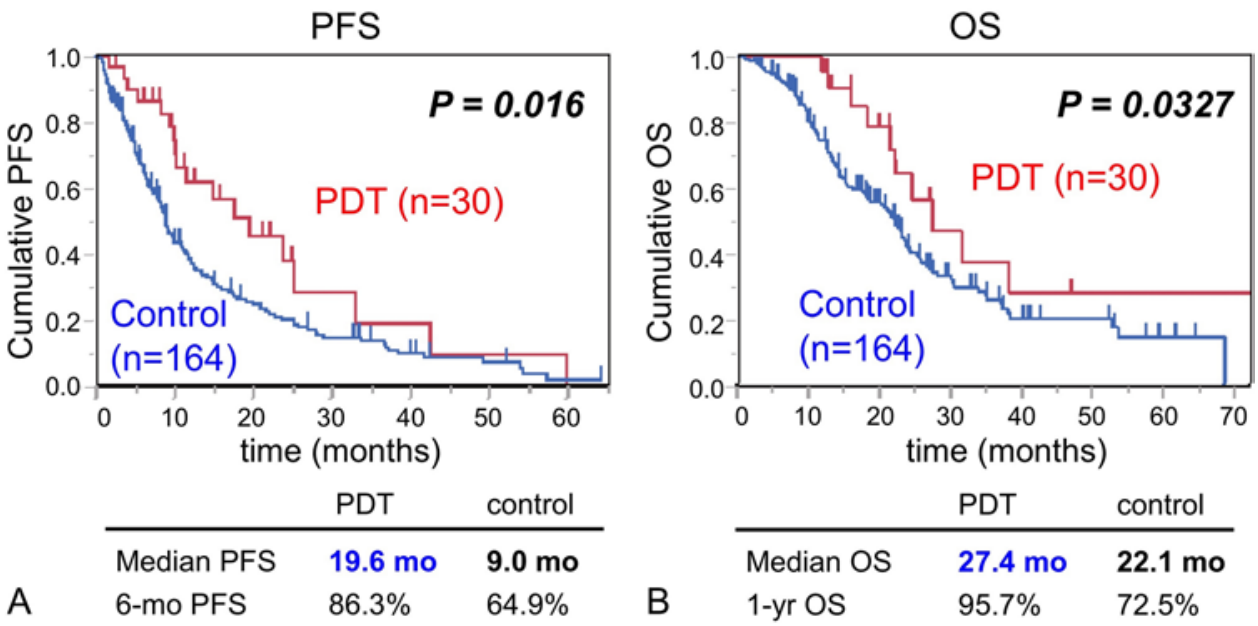

FIG. 1. Kaplan-Meier survival curves for PFS (A) and OS (B) in the PDT and control groups.

\section{Results of the Univariate and Multivariate Analyses}

Univariate analyses were conducted for the following parameters: age, preoperative KPS score, IDH mutation, PDT, and MIB-1 index. Among these factors, KPS score, IDH mutation, and PDT were identified as significant prognostic factors for PFS (Table 2); age, KPS score, $I D H$ mutation, and PDT were identified as significant prognostic factors for OS as well (Table 3). Multivariate analysis using the aforementioned factors revealed that PDT $(\mathrm{p}=$ 0.0023 and 0.0073$)$, KPS score ( $\mathrm{p}=0.0044$ and 0.0034$)$, and $I D H$ mutation ( $\mathrm{p}=0.0065$ and 0.032 ) were all significant independent prognostic factors for both PFS and OS (Tables 2 and 3). These results suggest that PDT might be a prognostic factor along with the other known prognostic factors, such as IDH mutation and KPS score.

\section{Pattern of Recurrence}

During the follow-up period, 18 of 30 patients in the PDT group experienced tumor recurrence. Interestingly, local recurrence was observed in only 10 patients (58.8\%), while 3 and 4 patients exhibited distant recurrence and dissemination, respectively (Table 4). On the other hand, 141 of 164 patients in the control group had recurrence, and $101(79.5 \%)$ had local recurrence. The PDT group showed a lower rate of local recurrence, although there was no statistical difference $(p=0.06$, Table 4$)$. Taken to-

TABLE 2. Univariate and multivariate analyses for PFS

\begin{tabular}{|c|c|c|c|c|c|c|}
\hline \multirow[b]{2}{*}{ Factor } & \multicolumn{3}{|c|}{ Univariate Analysis } & \multicolumn{3}{|c|}{ Multivariate Analysis } \\
\hline & $\mathrm{HR}$ & $\begin{array}{c}95 \% \\
\mathrm{Cl}\end{array}$ & $\begin{array}{c}\mathrm{p} \\
\text { Value }\end{array}$ & HR & $\begin{array}{c}95 \% \\
\mathrm{Cl}\end{array}$ & $\begin{array}{c}p \\
\text { Value }\end{array}$ \\
\hline $\begin{array}{c}\text { KPS score } \geq 80 \\
\text { vs }<80\end{array}$ & 0.53 & $0.37-0.77$ & 0.001 & 0.53 & $0.35-0.88$ & 0.0044 \\
\hline PDT vs no PDT & 0.55 & $0.32-0.88$ & 0.01 & 0.42 & $0.21-0.75$ & 0.0023 \\
\hline $\begin{array}{l}\text { IDH mutation } \\
\text { vs IDH } \\
\text { wildtype }\end{array}$ & 0.51 & $0.30-0.85$ & 0.008 & 0.27 & $0.27-0.82$ & 0.0065 \\
\hline $\begin{array}{c}\text { Age }<60 \text { vs } \\
\geq 60 \mathrm{yrs}\end{array}$ & 0.86 & $0.85-1.61$ & 0.34 & 0.80 & $0.55-1.18$ & 0.26 \\
\hline
\end{tabular}

gether, our results suggest that PDT provided excellent local control, thereby leading to increasing relative rates of distant recurrence and dissemination.

\section{Adverse Events and Radiological Changes After PDT}

Among the patients who received PDT, there were no severe adverse events of grade III or higher according to the CTCAE version 4.0. However, asymptomatic transient peripheral edema and fluid collection in the resection cavity were observed on MRI in 11 out of 30 patients (36.7\%). The fluid exhibited hyperintensity on T1-, T2- and FLAIRweighted images (Figs. 2E, 2F, 3E, and 3F). These MRI abnormalities completely disappeared within a few months (Figs. 2G, 2H, 3G, and 3H). In some patients, a linear contrast effect of the resected cavity wall on MRI appeared to last from several months to more than 1 year (Figs. 2I and 3I). However, this lesion showed faint uptake on methionine PET scans (Fig. 3J), and the effect was not considered a recurrence but an inflammatory change due to PDT.

\section{Representative Cases}

\section{Case 1}

A 62-year-old woman presented with weakness of her right lower limb and motor aphasia. MRI demonstrated

TABLE 3. Univariate and multivariate analyses for OS

\begin{tabular}{|c|c|c|c|c|c|c|}
\hline \multirow[b]{2}{*}{ Factor } & \multicolumn{3}{|c|}{ Univariate Analysis } & \multicolumn{3}{|c|}{ Multivariate Analysis } \\
\hline & $\mathrm{HR}$ & $\begin{array}{c}95 \% \\
\mathrm{Cl}\end{array}$ & $\begin{array}{c}p \\
\text { Value }\end{array}$ & $\mathrm{HR}$ & $\begin{array}{c}95 \% \\
\mathrm{Cl}\end{array}$ & $\begin{array}{c}\mathrm{p} \\
\text { Value }\end{array}$ \\
\hline $\begin{array}{l}\text { KPS score } \\
\quad \geq 80 \text { vs }<80\end{array}$ & 0.41 & $0.27-0.64$ & 0.0001 & 0.47 & $0.28-0.79$ & 0.0034 \\
\hline $\begin{array}{l}\text { PDT vs no } \\
\text { PDT }\end{array}$ & 0.50 & $0.24-0.91$ & 0.022 & 0.31 & $0.09-0.76$ & 0.0073 \\
\hline $\begin{array}{l}\text { IDH mutation } \\
\text { vs IDH } \\
\text { wildtype }\end{array}$ & 0.44 & $0.22-0.81$ & 0.007 & 0.50 & $0.24-0.95$ & 0.032 \\
\hline $\begin{array}{c}\text { Age }<60 \text { vs } \\
\geq 60 \mathrm{yrs}\end{array}$ & 0.65 & $0.44-0.95$ & 0.027 & 0.95 & $0.66-1.71$ & 0.8 \\
\hline
\end{tabular}


TABLE 4. Pattern of tumor recurrence

\begin{tabular}{lcc}
\hline \multicolumn{1}{c}{ Pattern } & PDT & Control \\
\hline Local & $10(58.8 \%)$ & $101(79.5 \%)$ \\
\hline Distant & 3 & 18 \\
\hline Dissemination & 4 & 8 \\
\hline Unknown & 1 & 14 \\
\hline Total & 18 & 141 \\
\hline
\end{tabular}

multiple lesions in the left frontal and parietal lobule. It also indicated that the lesions had low intensity on T1weighted images, ringlike enhancement on gadolinium uptake (Fig. 2A), and high intensity on FLAIR images (Fig. 2B). The enhanced lesions were removed using intraoperative MRI guidance, and a laser was used to irradiate to the cavity wall in a total of 6 different spots. Postoperatively, the patient still experienced right motor weakness and motor aphasia; the symptoms were unchanged compared to before the surgery. Postoperative MRI showed no residual enhanced lesions (Fig. 2C and D); 14-day postoperative MRI revealed fluid collection and edema (Fig. 2E and F), and these findings disappeared in 2 months (Fig. 2G and H). Pathological diagnosis of the tumor was glioblastoma, IDH wildtype, and the patient received fractionated radiation therapy $(60 \mathrm{~Gy})$ and concomitant temozolomide-based chemotherapy. She continued maintenance temozolomide-based chemotherapy for another 12 courses. At the latest follow-up, 28 months after the surgery, the patient showed no evidence of tumor recurrence and her KPS score was 60 (Fig. 2I and J).

\section{Case 2}

A 70-year-old man developed progressive motor aphasia and right hand fine motor dysfunction. MRI demonstrated a round mass lesion in the deep white matter in the left middle frontal gyrus involving the pyramidal tract. On MRI, the tumor exhibited low intensity on T1-weighted images, ringlike enhancement on gadolinium uptake (Fig. 3A), and mixed high intensity on FLAIR images (Fig. 3B). Gross-total removal of the enhanced lesion was performed using intraoperative MRI guidance (Fig. 3C and D), and the cavity wall was irradiated in a total of 4 spots. Postoperatively, the patient still exhibited mild right motor weakness and motor aphasia; the symptoms were unchanged compared to before the surgery. As in case 1,14-day postoperative MRI exhibited fluid collection and edema (Fig. $3 \mathrm{E}$ and $\mathrm{F}$ ), and these disappeared in 2 months (Fig. 3G and $\mathrm{H})$. The patient received fractionated radiation therapy (60 Gy) and concomitant temozolomide-based chemotherapy, followed by maintenance temozolomide-based chemotherapy for another 24 courses. A linear enhancement in the cavity wall appeared several months after the surgery, and it was present for more than 2 years (Fig. 3I). The lesion showed slight uptake in a methionine PET study with a tumor tissue/normal tissue ratio of 1.71 (Fig. 3J). At the latest follow-up, 30 months after the surgery, the patient showed no evidence of tumor recurrence and had a KPS score of 80 .

\section{Discussion}

PDT is a therapeutic method in which a photosensitizer (mainly a porphyrin derivative) administered in vivo is excited by laser light of a specific wavelength, and target cells
Before surgery
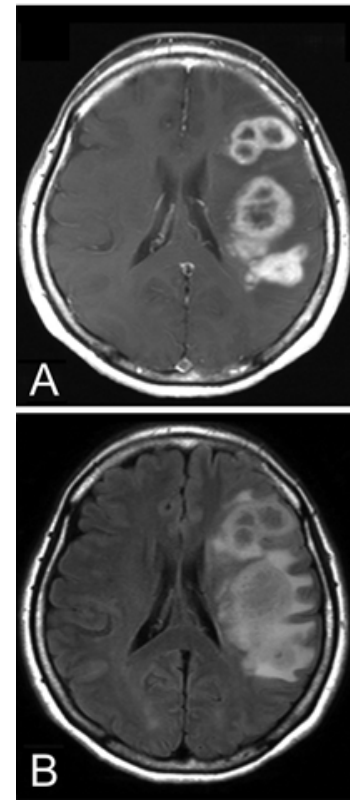

3 days
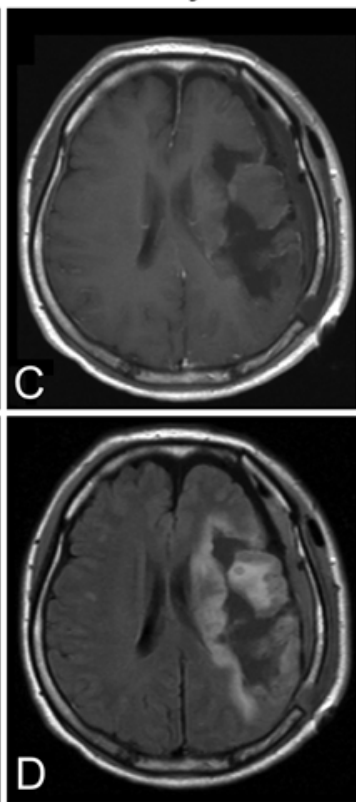

14 days
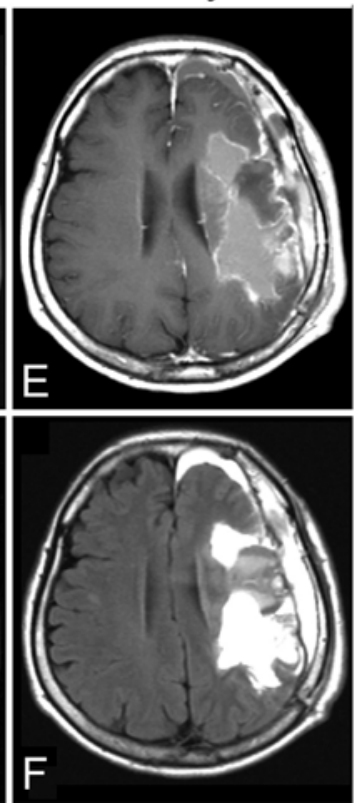

2 months
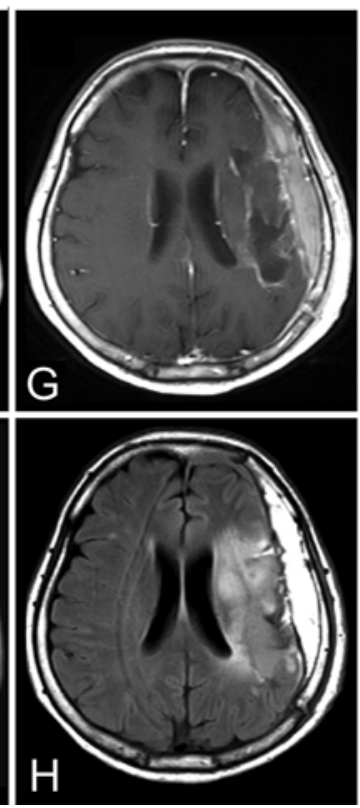

28 months

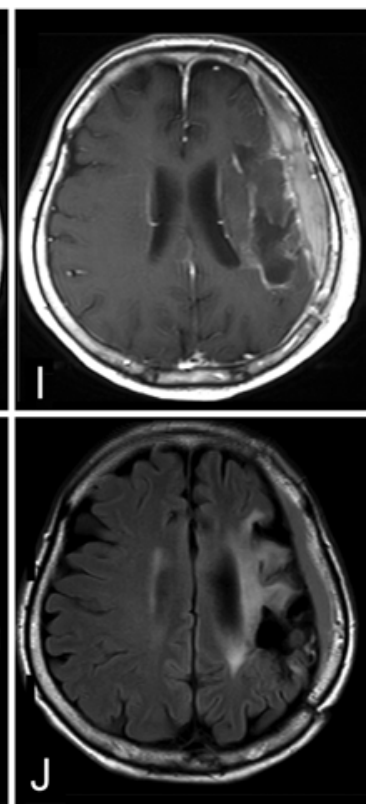

FIG. 2. Representative case 1. Preoperative contrast-enhanced T1-weighted (A) and FLAIR (B) MR images. Contrast-enhanced T1-weighted and FLAIR MRI findings 3 days ( $C$ and $D), 2$ weeks ( $E$ and $F), 2$ months ( $G$ and $H)$, and 28 months (I and $\mathbf{J})$ after the surgery. 

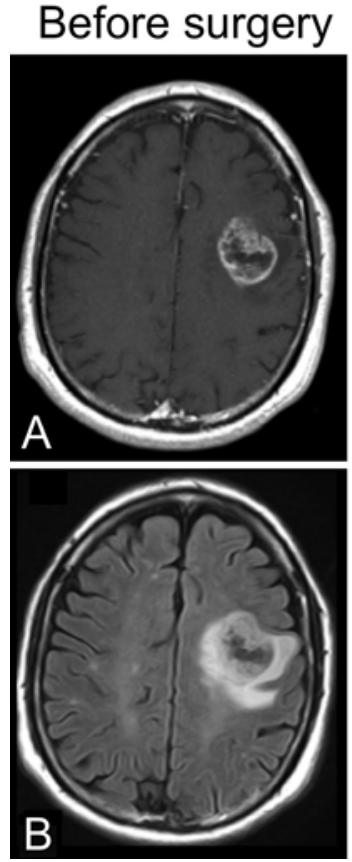

3 days
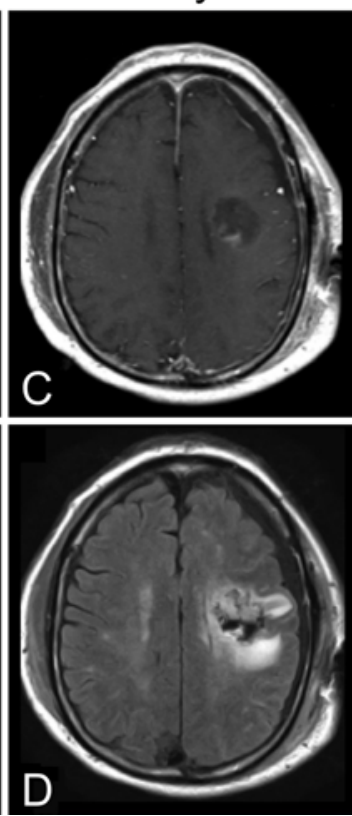

14 days
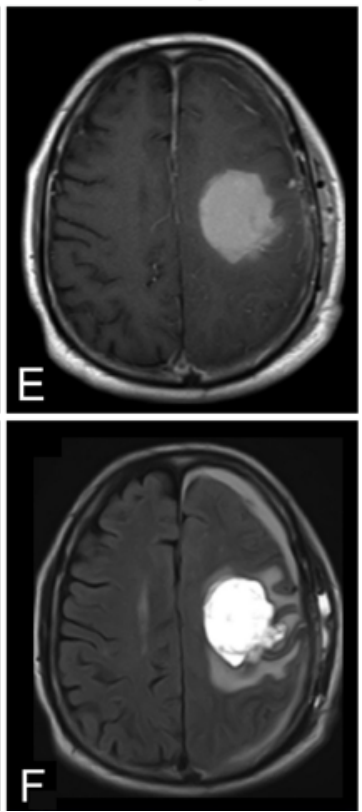

2 months
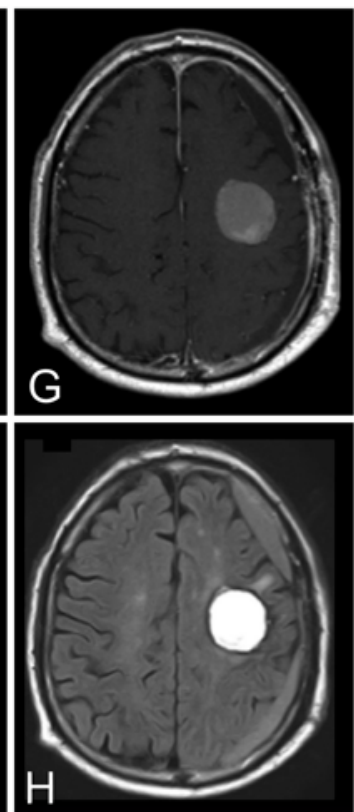

30 months
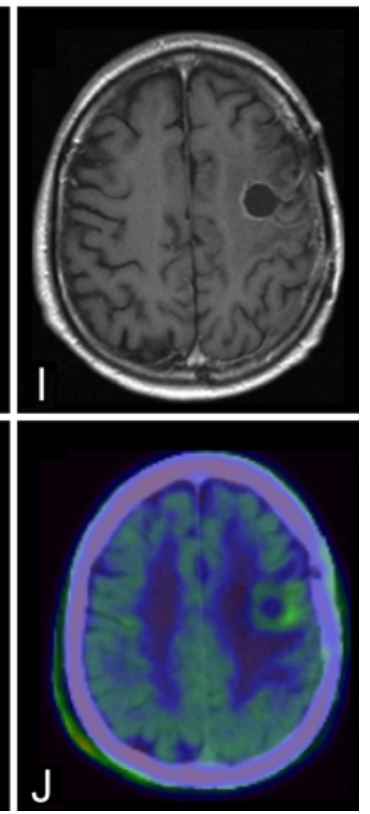

FIG. 3. Representative case 2. Preoperative contrast-enhanced T1-weighted (A) and FLAIR (B) MR images. Contrast-enhanced T1-weighted and FLAIR MRI findings 3 days ( $\mathbf{C}$ and D), 2 weeks ( $\mathbf{E}$ and $\mathbf{F}), 2$ months ( $\mathbf{G}$ and $\mathbf{H}$ ), and 30 months (I) after the surgery. PET study at 2 years postoperatively $(\mathrm{J})$.

are killed by active oxygen produced by a photochemical reaction. ${ }^{1,5}$ Photosensitizer administered in advance accumulates selectively in the tumor. ${ }^{25}$ When the target tissue is irradiated with laser light after the photosensitizer has accumulated in the target tissue, the photosensitizer is in an excited state. It is believed that when excited photosensitizer returns to the ground state, it reacts with oxygen in the tissues to generate active oxygen, especially singlet oxygen, which induces selective cell death of the target tissue. Both apoptosis and necrosis are thought to be involved as a mechanism of cell death. ${ }^{1,4,5}$ Since the migration distance of singlet oxygen is as short as $0.02-1 \mu \mathrm{m}$ and its lifetime is as short as $0.04-4 \mu \mathrm{sec},,^{12,22}$ there is no bystander effect on surrounding cells, and selective cell death of photosensitizer-accumulated tumor cells can be induced. By controlling the amount of laser light energy to be irradiated to such a degree that normal cells are not damaged, normal surrounding brain cells can be protected. It is also thought that there are antitumor effects due to the occlusion of microvessels ${ }^{4}$ and immunity induction effects. ${ }^{4,13}$

There are mainly two irradiation methods for PDT: one that is interstitial irradiation, and another that is outside irradiation to the wall of the removal cavity. Interstitial PDT requires implantation of light diffusers; the safety and effectiveness of interstitial PDT using aminolevulinic acid have been shown in both an experimental ${ }^{24}$ and a clini$\mathrm{cal}^{3,20}$ setting. On the other hand, cavity wall irradiation does not require a specific light diffuser but rather a laser irradiation tool. Stylli et al. have reported clinical results of PDT using a hematoporphyrin derivative for high-grade gliomas, and the median survival of newly diagnosed patients with glioblastoma was 14.3 months. ${ }^{23}$ Akimoto et al. have shown clinical results of PDT using talaporfin sodium for malignant gliomas. ${ }^{2}$ In both methods, the depth of laser light penetration is critical for successful PDT. In general, longer wavelengths of light will reach deeper into the tissue. The semiconductor laser for our study uses light with a wavelength of $664 \pm 2 \mathrm{~nm}$, and this is expected to penetrate into approximately $4-5 \mathrm{~mm}$ of tissue. However, it is possible to penetrate deeper into tumor tissue ${ }^{26}$ and into an edematous brain. ${ }^{25}$

PDT using talaporfin sodium has shown excellent clinical results in other cancers. Usuda et al. have shown a 90.4\% complete response rate in bronchogenic carcinomas in central airways with a diameter greater than $10 \mathrm{~mm}$ by using talaporfin sodium for the PDT. ${ }^{26}$ A multicenter phase II study of salvage PDT using talaporfin sodium for local failure after chemoradiotherapy or radiotherapy alone for esophageal cancer resulted in amazing clinical results with a $88.5 \%$ complete response rate, ${ }^{28}$ and the use of PDT for recurrent esophageal cancer was approved in Japan in 2015. In this study, we showed that PDT might provide excellent survival benefit for patients with newly diagnosed glioblastoma, as compared with treatment without PDT. A survival advantage for PDT was shown in both univariate and multivariate analyses, along with representative prognostic markers for glioblastoma such as $I D H$ mutation and better KPS scores. This favorable result warrants future controlled randomized clinical trials on PDT. Moreover, this result strongly supports the idea that local control is important for the management of glioblastoma, despite its infiltrative nature. After maximum tumor resection, a number of invading tumor cells may still exist in the peripheral normal brain; however, the cell density decreases with increased distance. Therefore, PDT may selectively kill infiltrating tumor cells at a high density, while protecting normal cells, thereby providing efficient cytoreduction and, eventually, prolonged tumor control. In fact, the rate 
of local recurrence was relatively low in the PDT group in the present study (Table 4). Given the significantly longer PFS time in the PDT group, PDT seems to prolong patient survival by improving the local control. It is also possible that not only the local tumor control but also the systemic immunoreaction activated by PDT may contribute to better prognosis. ${ }^{4,13}$ It is suggested that the PDT-treated dying cells and tumor antigens may activate various immunoreactions, including antigen presentation of dendritic cells and the recruitment of antigen-specific cytotoxic T-lymphocytes., ${ }^{4,13}$ Transient peripheral edema and fluid collection in the resection cavity after PDT observed in our case series may be the evidence of an immunoreaction after PDT.

Since PDT using talaporfin sodium and a semiconductor laser for malignant brain tumors is a challenging treatment method, we limited the number of laser irradiations to up to 3 spots in our previous phase II clinical study. ${ }^{14}$ In that study, the median PFS was 12.0 months and the local PFS - that is, the PFS of the laser-irradiated site-was 19.6 months. ${ }^{14}$ Given the finding that there were almost no severe adverse effects caused by PDT in the phase II clinical study, there was no limitation in the number of irradiation spots in our subsequent study, and we irradiated more spots, ranging from 3-13 locations, after the approval of this technology. Interestingly, the median PFS (19.6 months) in the patients treated with multiple laser irradiations was identical to the local PFS among the patients in the phase II clinical study. ${ }^{14}$ This suggests that we may be able to prevent local tumor recurrence from nonirradiated sites by using multiple irradiations.

The major limitation of this new treatment is the limited effects of laser irradiation on deep-seated tumors and cases involving a narrow surgical window. Because the PDT laser unit is attached to a microscope and because the laser irradiation proceeds linearly from the optical source of the microscope, efficient irradiation is difficult in such cases. Therefore, novel irradiation devices, such as the endoscopic laser unit that is currently used for esophageal and lung cancers, should be utilized, with modification, for glioblastoma. Although the PDT-treated patients showed excellent local tumor control, many patients eventually experienced distant recurrences and CSF dissemination. We have to emphasize that extensive systemic treatments, in addition to the PDT, are necessary for long-term tumor control. The other limitations of this study include the limited number of patients and the single-institute study design. To further demonstrate the efficacy of PDT with talaporfin sodium and a semiconductor laser for newly diagnosed glioblastoma, a controlled phase III clinical trial with a sufficient number of patients should be performed. The most scientifically accurate clinical trial design is a comparison of the four groups-talaporfin and laser, talaporfin only, laser alone, and no treatment-but it has already been shown that talaporfin alone and laser alone have no antitumor effect in basic experiments. Therefore, we plan to compare two groups: a PDT group and a surgery-alone group.

\section{Conclusions}

The present study showed a statistically better prognosis in the patients with newly diagnosed glioblastoma treated with PDT than those treated without PDT. In addition to excellent local control, this study also demonstrated the safety of multiple laser irradiations to more than 10 spots, although asymptomatic transient MRI abnormalities were observed in some patients. This completely new concept for treatment may become a new standard treatment for glioblastoma. A randomized controlled clinical trial for the PDT is warranted.

\section{Acknowledgments}

We thank Mr. Takashi Sakayori and Ms. Asuka Komori (Tokyo Women's Medical University) for their assistance in the neuropathological study.

\section{References}

1. Akimoto J: Photodynamic therapy for malignant brain tumors. Neurol Med Chir (Tokyo) 56:151-157, 2016

2. Akimoto J, Haraoka J, Aizawa K: Preliminary clinical report on safety and efficacy of photodynamic therapy using talaporfin sodium for malignant gliomas. Photodiagn Photodyn Ther 9:91-99, 2012

3. Beck TJ, Kreth FW, Beyer W, Mehrkens JH, Obermeier A, Stepp H, et al: Interstitial photodynamic therapy of nonresectable malignant glioma recurrences using 5-aminolevulinic acid induced protoporphyrin IX. Lasers Surg Med 39:386-393, 2007

4. Castano AP, Mroz P, Hamblin MR: Photodynamic therapy and anti-tumour immunity. Nat Rev Cancer 6:535-545, 2006

5. Dolmans DE, Fukumura D, Jain RK: Photodynamic therapy for cancer. Nat Rev Cancer 3:380-387, 2003

6. Fukui A, Muragaki Y, Saito T, Maruyama T, Nitta M, Ikuta $\mathrm{S}$, et al: Volumetric analysis using low-field intraoperative magnetic resonance imaging for 168 newly diagnosed supratentorial glioblastomas: effects of extent of resection and residual tumor volume on survival and recurrence. World Neurosurg 98:73-80, 2017

7. Kato H, Furukawa K, Sato M, Okunaka T, Kusunoki Y, Kawahara M, et al: Phase II clinical study of photodynamic therapy using mono-L-aspartyl chlorin e6 and diode laser for early superficial squamous cell carcinoma of the lung. Lung Cancer 42:103-111, 2003

8. Konishi Y, Muragaki Y, Iseki H, Mitsuhashi N, Okada Y: Patterns of intracranial glioblastoma recurrence after aggressive surgical resection and adjuvant management: retrospective analysis of 43 cases. Neurol Med Chir (Tokyo) 52:577-586, 2012

9. Li YM, Suki D, Hess K, Sawaya R: The influence of maximum safe resection of glioblastoma on survival in 1229 patients: Can we do better than gross-total resection? J Neurosurg 124:977-988, 2016

10. Louis DN, Ohgaki H, Wiestler OD, Cavenee WK, Burger PC, Jouvet A, et al: The 2007 WHO classification of tumours of the central nervous system. Acta Neuropathol 114:97-109, 2007

11. Mimura S, Ito Y, Nagayo T, Ichii M, Kato H, Sakai H, et al: Cooperative clinical trial of photodynamic therapy with photofrin II and excimer dye laser for early gastric cancer. Lasers Surg Med 19:168-172, 1996

12. Moan J, Berg K: The photodegradation of porphyrins in cells can be used to estimate the lifetime of singlet oxygen. Photochem Photobiol 53:549-553, 1991

13. Mroz P, Hashmi JT, Huang YY, Lange N, Hamblin MR: Stimulation of anti-tumor immunity by photodynamic therapy. Expert Rev Clin Immunol 7:75-91, 2011

14. Muragaki Y, Akimoto J, Maruyama T, Iseki H, Ikuta S, Nitta 
M, et al: Phase II clinical study on intraoperative photodynamic therapy with talaporfin sodium and semiconductor laser in patients with malignant brain tumors. J Neurosurg 119:845-852, 2013

15. Murakami R, Hirai T, Nakamura H, Furusawa M, Nakaguchi Y, Uetani H, et al: Recurrence patterns of glioblastoma treated with postoperative radiation therapy: relationship between extent of resection and progression-free interval. Jpn J Radiol 30:193-197, 2012

16. Muroya T, Kawasaki K, Suehiro Y, Kunugi T, Umayahara K, Akiya T, et al: Application of PDT for uterine cervical cancer. Diagn Ther Endosc 5:183-190, 1999

17. Quirk BJ, Brandal G, Donlon S, Vera JC, Mang TS, Foy $\mathrm{AB}$, et al: Photodynamic therapy (PDT) for malignant brain tumors-where do we stand? Photodiagn Photodyn Ther 12:530-544, 2015

18. Sanai N, Polley MY, McDermott MW, Parsa AT, Berger MS: An extent of resection threshold for newly diagnosed glioblastomas. J Neurosurg 115:3-8, 2011

19. Senft C, Bink A, Franz K, Vatter H, Gasser T, Seifert V: Intraoperative MRI guidance and extent of resection in glioma surgery: a randomised, controlled trial. Lancet Oncol 12:997-1003, 2011

20. Stummer W, Beck T, Beyer W, Mehrkens JH, Obermeier A, Etminan N, et al: Long-sustaining response in a patient with non-resectable, distant recurrence of glioblastoma multiforme treated by interstitial photodynamic therapy using 5-ALA: case report. J Neurooncol 87:103-109, 2008

21. Stupp R, Mason WP, van den Bent MJ, Weller M, Fisher $\mathrm{B}$, Taphoorn MJ, et al: Radiotherapy plus concomitant and adjuvant temozolomide for glioblastoma. $\mathbf{N}$ Engl J Med 352:987-996, 2005

22. Stylli SS, Kaye AH: Photodynamic therapy of cerebral glioma-a review Part I-a biological basis. J Clin Neurosci 13:615-625, 2006

23. Stylli SS, Kaye AH, MacGregor L, Howes M, Rajendra P: Photodynamic therapy of high grade glioma-long term survival. J Clin Neurosci 12:389-398, 2005

24. Tetard MC, Vermandel M, Leroy HA, Leroux B, Maurage CA, Lejeune JP, et al: Interstitial 5-ALA photodynamic therapy and glioblastoma: Preclinical model development and preliminary results. Photodiagn Photodyn Ther 13:218224,2016

25. Tsurubuchi T, Zaboronok A, Yamamoto T, Nakai K, Yoshida
F, Shirakawa M, et al: The optimization of fluorescence imaging of brain tumor tissue differentiated from brain edema-in vivo kinetic study of 5-aminolevulinic acid and talaporfin sodium. Photodiagn Photodyn Ther 6:19-27, 2009

26. Usuda J, Ichinose S, Ishizumi T, Hayashi H, Ohtani K, Maehara $S$, et al: Outcome of photodynamic therapy using NPe6 for bronchogenic carcinomas in central airways $>1.0 \mathrm{~cm}$ in diameter. Clin Cancer Res 16:2198-2204, 2010

27. Wen PY, Macdonald DR, Reardon DA, Cloughesy TF, Sorensen AG, Galanis E, et al: Updated response assessment criteria for high-grade gliomas: response assessment in neuro-oncology working group. J Clin Oncol 28:1963-1972, 2010

28. Yano T, Kasai H, Horimatsu T, Yoshimura K, Teramukai S, Morita S, et al: A multicenter phase II study of salvage photodynamic therapy using talaporfin sodium (ME2906) and a diode laser (PNL6405EPG) for local failure after chemoradiotherapy or radiotherapy for esophageal cancer. Oncotarget 8:22135-22144, 2017

\section{Disclosures}

The authors report no conflict of interest concerning the materials or methods used in this study or the findings specified in this paper.

\section{Author Contributions}

Conception and design: Muragaki, Nitta. Acquisition of data: Nitta, Maruyama, Komori, Ikuta, Saito, Yasuda, Hosono, Okamoto, Koriyama. Analysis and interpretation of data: Nitta, Komori, Ikuta, Yasuda, Hosono, Okamoto, Koriyama. Drafting the article: Nitta. Critically revising the article: Muragaki, Nitta. Reviewed submitted version of manuscript: Muragaki, Nitta, Maruyama, Saito. Approved the final version of the manuscript on behalf of all authors: Muragaki. Statistical analysis: Nitta, Ikuta. Administrative/technical/material support: Iseki, Kawamata. Study supervision: Muragaki, Kawamata.

\section{Correspondence}

Yoshihiro Muragaki: Tokyo Women's Medical University, Shinjuku-ku, Tokyo, Japan.ymuragaki@twmu.ac.jp. 